ARTICLE

\title{
Quantitative operando visualization of the energy band depth profile in solar cells
}

\author{
Qi Chen ${ }^{1,2}$, Lin Mao ${ }^{1}$, Yaowen $\mathrm{Li}^{1,3}$, Tao Kong ${ }^{4}, \mathrm{Na} \mathrm{Wu}{ }^{5}$, Changqi $\mathrm{Ma}^{5}$, Sai Bai ${ }^{6}$, Yizheng Jin 6 , \\ Dan Wu1, Wei Lu1, Bing Wang ${ }^{2} \&$ Liwei Chen ${ }^{1}$
}

The energy band alignment in solar cell devices is critically important because it largely governs elementary photovoltaic processes, such as the generation, separation, transport, recombination and collection of charge carriers. Despite the expenditure of considerable effort, the measurement of energy band depth profiles across multiple layers has been extremely challenging, especially for operando devices. Here we present direct visualization of the surface potential depth profile over the cross-sections of operando organic photovoltaic devices using scanning Kelvin probe microscopy. The convolution effect due to finite tip size and cantilever beam crosstalk has previously prohibited quantitative interpretation of scanning Kelvin probe microscopy-measured surface potential depth profiles. We develop a bias voltage-compensation method to address this critical problem and obtain quantitatively accurate measurements of the open-circuit voltage, built-in potential and electrode potential difference.

\footnotetext{
${ }^{1} i$-Lab, Suzhou Institute of Nano-Tech and Nano-Bionics, Chinese Academy of Sciences, Suzhou 215123, China. ${ }^{2}$ Hefei National Laboratory for Physical Sciences at the Microscale and Synergetic Innovation Center of Quantum Information \& Quantum Physics, University of Science and Technology of China, Hefei 230026, China. ${ }^{3}$ Advanced Opto-electronic Materials Lab of Soochow University, Department of Polymer Science and Engineering, College of Chemistry, Chemical Engineering and Materials Science, Soochow University, Suzhou 215123, China. ${ }^{4}$ Division of Nanobiomedicine, Suzhou Institute of Nano-Tech and Nano-Bionics, Chinese Academy of Sciences, Suzhou 215123, China. ${ }^{5}$ Division of Printed Electronics, Suzhou Institute of Nano-Tech and Nano-Bionics, Chinese Academy of Sciences, Suzhou 215123, China. ${ }^{6}$ State Key Laboratory of Silicon Materials, Department of Materials Science and Engineering, Zhejiang University, Hangzhou 310027, China. Correspondence and requests for materials should be addressed to L.C. (email: Iwchen2008@sinano.ac.cn).
} 
$\mathrm{T}$ he energy band alignment across multiple layers is extremely important for solar cells because elementary photovoltaic processes, such as charge separation, carrier transport and collection ${ }^{1-4}$, as well as undesirable recombination ${ }^{5,6}$, all depend on this alignment. However, experimental determination of the energy-level alignment and the quantitative measurement of related parameters, such as the built-in potential $\left(V_{\mathrm{bi}}\right)$, have been difficult to realize, especially under typical operating conditions for the devices. Indirect methods, such as Mott-Schottky analysis ${ }^{7-10}$, electroabsorption spectroscopy ${ }^{1-13}$ and dark current density-voltage $(J-V)$ curve fitting ${ }^{9,14}$, have been applied to measure the $V_{b i}$ in solar cells. Scanning Kelvin probe microscopy (SKPM), a functional imaging technique that maps the local surface potential (SP) of a sample ${ }^{15,16}$, has also been employed to measure the potential difference between adjacent layers in solar cells ${ }^{17-20}$.

Recently, SKPM has been used to image cross-sections of solar cells to probe the SP depth profile across multiple layers ${ }^{21-26}$. The vacuum level (VL) in an energy band structure is defined as the energy of an electron resting right at the surface of a material; thus, the VL alignment within a device can be obtained by multiplying the SP depth profile by a constant, that is, the electron charge $e^{27,28}$. In principle, this approach offers the advantage of being able to directly visualize the energy band alignment across the device. However, a major obstacle has limited the application of this approach in solar cell research: quantitatively accurate measurements and interpretations are yet to be realized in cross-sectional SKPM studies. Critical parameters for the operating mechanism of solar cells, such as the open-circuit voltage $\left(V_{\mathrm{oc}}\right)$ and $V_{\mathrm{bi}}$, are often found to be much smaller than expected from $J-V$ characterization ${ }^{21-23}$. Overall, the results obtained from cross-sectional SKPM measurements are often uncorrelated with the device performance and $J-V$ characteristics, and are thus not applicable for understanding practical devices in their actual operating states.

In this study, we address the challenges in quantitative SKPM potentiometry using high-efficiency organic photovoltaic (OPV) cells $^{29-31}$ as model systems. As shown in Fig. 1a, cross-sections of OPV devices are exposed by using ion-beam milling, and the SP depth profiles of the OPV device under operating conditions are directly visualized using SKPM imaging on the cross-section
(Fig. 1b). The tip/cantilever convolution effect due to finite tip size and cantilever beam crosstalk is identified as the source of the systematic underestimate of SP differences in cross-sectional SKPM measurements ${ }^{32-34}$. A bias compensation method is developed to obtain quantitative measurements of internal potential difference parameters, such as $V_{\mathrm{oc}}$, electrode potential difference $\left(V_{\mathrm{td}}\right)$ and $V_{\mathrm{bi}}$, in OPV devices.

\section{Results}

Preparation of OPV device cross-sections. The OPV devices are fabricated on the glass substrate using a conventional stacking of indium tin oxide (ITO)/ $\mathrm{MoO}_{x} /$ active materials/LiF/Al. To prepare a smooth cross-section, the device was cut from the back of the glass substrate. The exposed edge is then milled under an $\mathrm{Ar}^{+}$beam (Ilion ${ }^{+}$693, Gatan Inc., Pleasanton, CA, USA; Fig. 1a). The device fabrication and cross-section preparation are described in details in the Methods section. Figure 1c displays the scanning electron microscopy (SEM)-backscattered electron image of the cross-section. The contrast in the image primarily results from materials with different atomic numbers rather than the topography. The cross-section morphology is further confirmed using X-ray energy dispersive spectrum mapping in Supplementary Fig. 1. J-V curves of a typical bulk heterojunction (BHJ) device that is obtained using poly(3-hexylthiophene) (P3HT):[6,6]-phenyl- $\mathrm{C}_{61}$-butyric acid methyl ester (PCBM)active materials exhibit a power conversion efficiency (PCE) of 3.47\% (Fig. 1d, Table 1). The same device exhibits PCE of 3.17\% after cross-section ion milling and $2.65 \%$ after SKPM imaging with very stable $V_{\text {oc. }}$ Cross-sectioning was shown to result in small drops in $J_{\mathrm{sc}}(\sim 6.98 \%$ drop$)$ and fill factor (FF; $\sim 3.23 \%$ drop), while the SKPM imaging resulted in further decreases in $J_{\mathrm{sc}}$ ( $\sim 11.23 \%$ drop) and FF ( $\sim 4.84 \%$ drop). Although the chemical changes associated with these performance decreases may, in principle, be significant, we believe that the cross-sectional SP mapping retains useful qualitative information about the pristine devices because the device $V_{\text {oc }}$ is well maintained throughout the cross-sectioning and measurement processes. Since the device is exposed to the ambient environment during the ion-beam milling, the device stability under ambient condition and the conditions of SKPM measurements is highly important. Note that, in
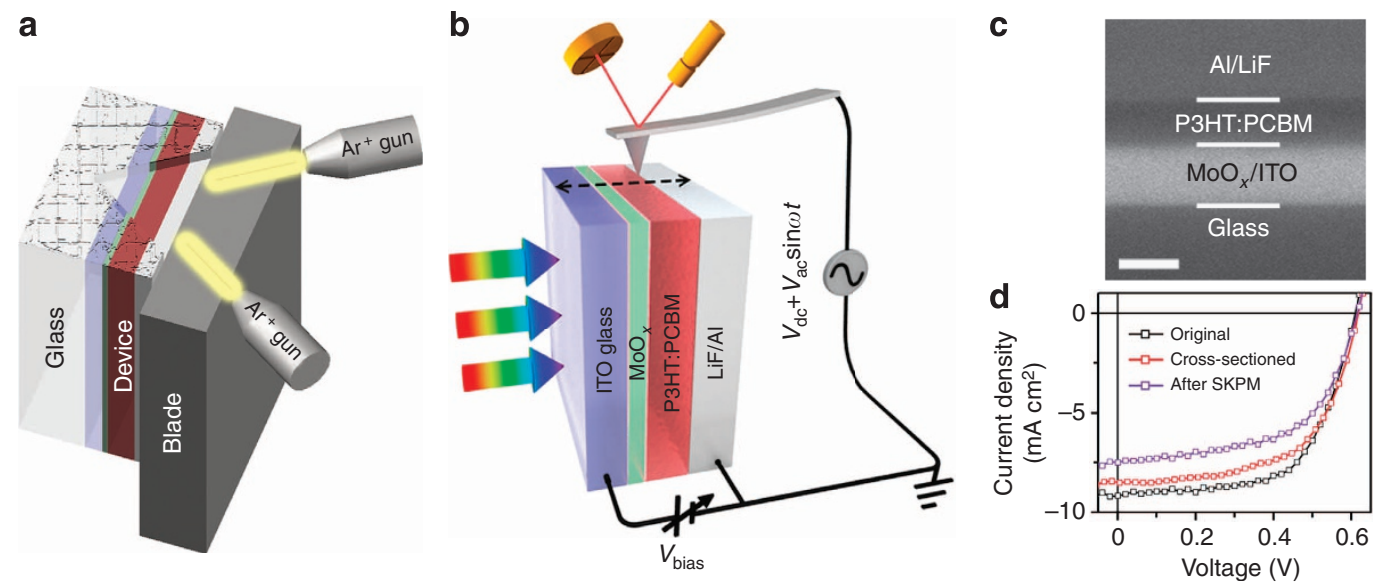

Figure 1 | Operando probing of solar cell energy band depth profiles with cross-sectional SKPM. (a) Schematic illustration of ion-beam-milling configuration to expose a smooth cross-section of an OPV device; (b) schematic illustration of cross-sectional SKPM measurements under operating conditions such as illumination and bias voltages. The cantilever approaches the sample from the side opened up using ion-beam milling. SKPM scanning is carried out with the cantilever long axis normal to the device plane; (c) SEM micrograph of an ion-beam-milled OPV device cross-section (scale bar, $250 \mathrm{~nm}$ ); and (d) current density versus voltage $(\mathrm{J}-\mathrm{V})$ characteristics of an OPV device with a conventional configuration ITO/MoO $/ \mathrm{P3HT:PCBM/LiF/AI}$ under AM 1.5G illumination before (black) and after (red) cross-section preparation, and after SKPM measurement (voilet), indicating good device stability. 
this study, it is critical to replace the typical poly(3,4-ethylenedioxythiophene):poly(styrenesulfonic acid) (PEDOT:PSS) anode interlayer with $\mathrm{MoO}_{x}$ because PEDOT:PSS is detrimental to the device stability ${ }^{35}$. Supplementary Fig. 2 shows that this device maintained $\simeq 68 \%$ of its original PCE after $100 \mathrm{~h}$ of exposure to the ambient environment, and the drop in efficiency over the first $40 \mathrm{~h}$ is only $\sim 2 \%$. In contrast, the devices with PEDOT:PSS interlayers decay much faster and completely lose their photovoltaic properties in $\simeq 80 \mathrm{~h}$. The successful preparation of the cross-section and maintenance of device stability during SKPM measurements enable us to investigate the energy band profiles in operando devices.

Qualitative energy band alignment in operando BHJ devices. Figure $2 a, c$ is topographical and phase images, respectively, of the cross-sections that are obtained via atomic force microscopy (AFM). The morphology of the ion-milled cross-section is

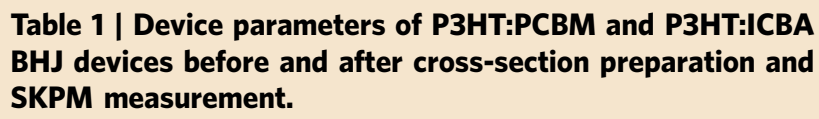

\begin{tabular}{llcccc}
$\begin{array}{l}\text { Active } \\
\text { layer }\end{array}$ & Device & $\mathbf{V}_{\text {oc }}(\mathbf{V})$ & $\begin{array}{c}\mathbf{J}_{\text {sc }} \\
\mathbf{( m A} \mathbf{~ c m}\end{array}$ & FF & PCE (\%) \\
\hline P3HT:PCBM & Original & 0.61 & 9.17 & 0.62 & 3.47 \\
& Cross-sectioned & 0.62 & 8.53 & 0.60 & 3.17 \\
& After SKPM & 0.62 & 7.50 & 0.57 & 2.65 \\
P3HT:ICBA & Original & 0.84 & 9.41 & 0.62 & 4.90 \\
& Cross-sectioned & 0.84 & 8.96 & 0.59 & 4.44 \\
& After SKPM & 0.84 & 7.79 & 0.56 & 3.66 \\
\hline
\end{tabular}

$\mathrm{BHJ}$, bulk heterojunction; $\mathrm{FF}$, fill factor; $I C B A$, indene- $\mathrm{C}_{60}$ bisadduct; $\mathrm{PCBM},[6,6]$-phenyl- $\mathrm{C}_{61}$ butyric acid methyl ester; P3HT, poly(3-hexylthiophene); SKPM, Scanning Kelvin probe microscopy. relatively smooth with an $\simeq 10$-nm-height difference between layers (Fig. 2b). A sharp contrast in the phase channel (Fig. 2d) is resulted from different mechanical properties of the organic and inorganic layers and is used to identify the interfaces within the device.

In equilibrium states, the Fermi levels are aligned across any interfaces. In OPV devices, the difference in the work function of the materials leads to VL offsets across different layers. However, the physical and chemical interactions between different materials in contact with each other may result in the formation of interfacial electronic states, interfacial charge transfer and the occupation of interfacial states, producing complicated interfacial dipole moments and band-bending 7,9,20,27,28,36. Thus, energy band alignment in devices is difficult to predict and has not been quantitatively measured in experiments so far. Figure $2 \mathrm{e}, \mathrm{f}$ presents SP images of the device cross-section acquired with an amplitude-modulation (AM) mode SKPM under open-circuit conditions in the dark and under AM $1.5 \mathrm{G}$ illumination, respectively. The SP depth profile across the device (Fig. $2 \mathrm{~g}$ ) is obtained by averaging at least 10 scan lines in the slow-scan direction. The SP profile under short-circuit conditions in the dark is also shown and essentially overlaps with the open-circuit SP profile. The depth profiles in Fig. $2 \mathrm{~g}$ show two important qualitative features: first, the potential drop is distributed throughout the entire active layer and, second, the SP decreases more rapidly at the cathodic $(\mathrm{LiF} / \mathrm{Al})$ interface than at the anodic $\left(\mathrm{MoO}_{x} / \mathrm{ITO}\right)$ interface (confirmed by the derivative of the SP profile as shown in Supplementary Fig. 3). These results can be explained using the energy band diagrams that are shown in Fig. 3. As the different layers (Fig. 3a) are fully in contact within the device, Fermi-level alignment is established irrespective of whether the configuration is a short-circuit or an open-circuit in the dark. The difference in the material work function is then reflected in the variation in the VL across the layers (Fig. 3b). It

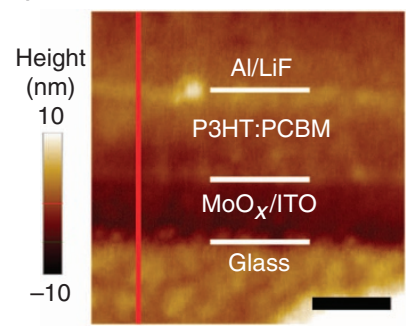

b

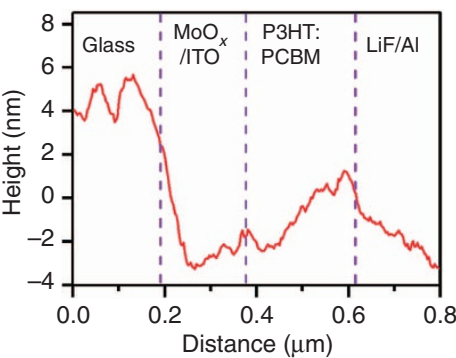

C

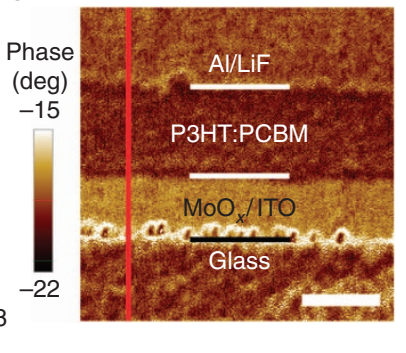

d

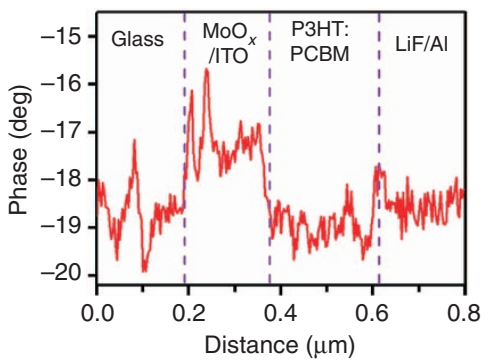

e

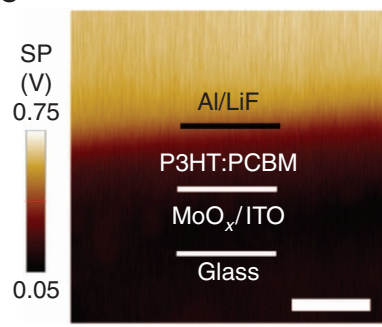

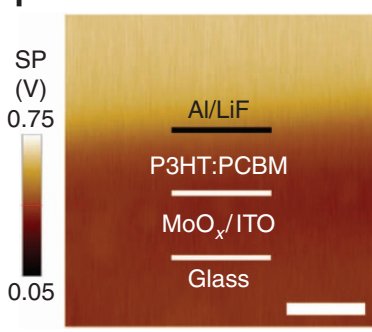

g

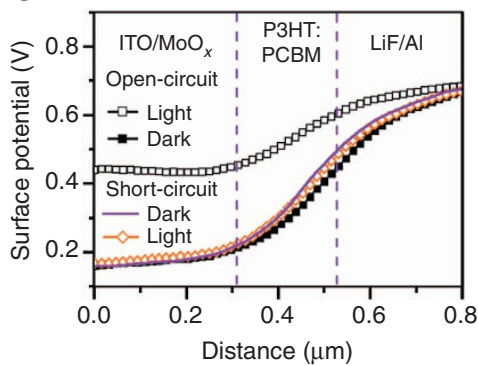

h

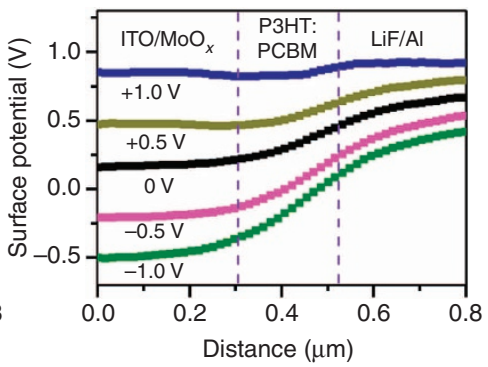

Figure 2 | Cross-sectional images and depth profiles of a BHJ device. (a) Topography and (c) phase images of the ITO/MoO $/$ P3HT:PCBM/LiF/AI device cross-section obtained with AFM (scale bars, $200 \mathrm{~nm}$ ). The line profiles in $\mathbf{b}, \mathbf{d}$ correspond to the red lines in $\mathbf{a}, \mathbf{c}$, respectively. SP images of the P3HT:PCBM $\mathrm{BHJ}$ device in open-circuit (e) in the dark and (f) under AM 1.5G illumination (scale bar, $250 \mathrm{~nm}$ ); (g) SP depth profiles of the device in open-circuit in the dark (solid black squares), in open-circuit under AM $1.5 \mathrm{G}$ illumination (open black squares), in short-circuit in the dark (solid purple line) and in short-circuit under AM $1.5 \mathrm{G}$ illumination (open orange diamonds); (h) SP depth profiles of the P3HT:PCBM BHJ device in the dark with bias voltages ranging from -1.0 to $+1.0 \mathrm{~V}$. The wiring configuration for applying the bias voltage is illustrated in Fig. $1 \mathrm{~b}$. 
a

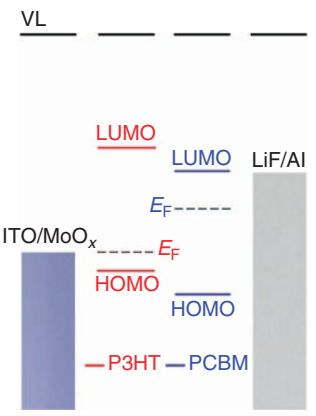

b
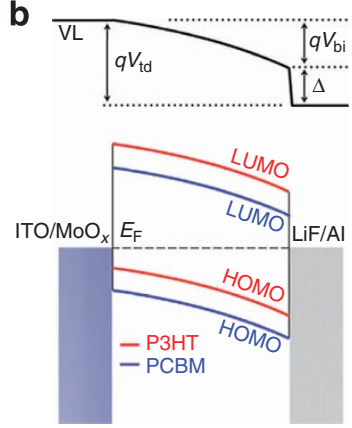

c
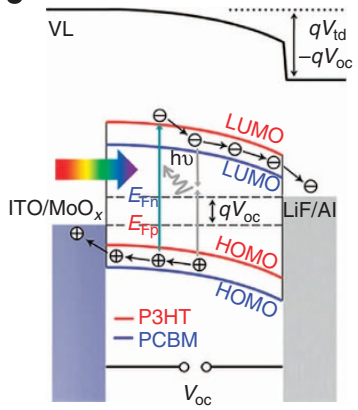

d
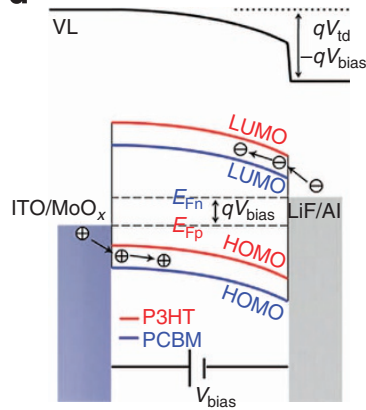

Figure 3 I Device energy band diagram under different conditions. (a) VL alignment of an ITO/MoO $/$ P3HT:PCBM/LiF/AI BHJ device before contact; (b) Fermi-level alignment after contact in the dark; (c) separated quasi-Fermi levels for electrons and holes under illumination; and (d) separated quasi-Fermi levels due to bias voltages between the two electrodes. Here, HOMO means highest occupied molecular orbital and LUMO means lowest unoccupied molecular orbital.

has been reported that $\mathrm{P} 3 \mathrm{HT}$ is enriched at the anodic interface because of vertical phase segregation within the $\mathrm{BHJ}^{37,38}$. The work function of $\mathrm{P} 3 \mathrm{HT}$ is very close to that of the anode; thus, the VL shift at the anodic interface is negligible, and the change in the SP at the anodic side is rather slow. At the cathodic side, the work function offset between LiF/Al and the P3HT:PCBM mixture is compensated for by an interface state-induced dipole moment and band bending within the active layer to achieve the Fermi-level alignment. The interface states may arise from physical and chemical interactions between the organic material and the metal electrode ${ }^{7}$. The low work function of $\mathrm{LiF} / \mathrm{Al}$ can result in the injection of electrons from the metal electrode to the interface states. These localized charged states form an electrical dipole moment that causes a sharp VL shift at the cathodic interface, $\Delta$. However, the density of interface states in organic materials is typically much lower than that in inorganic semiconductors, and the corresponding interfacial dipole is not sufficiently strong to fully compensate for the work function difference. Therefore, the $\mathrm{LiF} / \mathrm{Al}$ electrode injects more electrons into the P3HT:PCBM BHJ, resulting in band bending $\left(q V_{\mathrm{bi}}\right)$ in the active layer. This picture explains the continuous potential drop across the entire active layer and the rapid potential change at the cathode interface. Overall, the VL shift at the cathode interface $(\Delta)$ and the band bending $\left(\mathrm{q} V_{\mathrm{bi}}\right)$ in the active layer add up to the total energy drop from the anode to the cathode ( $q V_{\mathrm{td}}$; Fig. 3b).

The SP profile exhibits considerable differences under illumination in the open-circuit configuration (Fig. 2g). The total SP drop from the cathode to the anode, $V_{\mathrm{td}}$, is reduced under illumination in the open-circuit configuration. This result is obtained because of the accumulation of photo-generated carriers at the two electrodes, which establishes the quasi-Fermi levels of the hole $\left(E_{\mathrm{Fp}}\right)$ and the electron $\left(E_{\mathrm{Fn}}\right)$ at the anode and cathode, respectively. The direction of the field generated by the accumulated photo-generated carriers is opposite to that of the built-in field; therefore, the overall VL drop across the device under open-circuit conditions and illumination is less than that for the corresponding case in the dark (Fig. 3c).

The observed SP profile of the operando device, which is optimized with a P3HT:PCBM-active layer thickness of $\simeq 200 \mathrm{~nm}$, is consistent with the device performance. The continuous potential drop indicates the existence of a built-in electric field across the entire BHJ. The large donor-acceptor interface area in the bi-continuous $\mathrm{BHJ}$ facilitates the dissociation of photo-generated excitons into geminate carrier pairs; the builtin field further separates the geminate pairs and prevents geminate recombination ${ }^{5,6}$, resulting in a high short-circuit current density $\left(J_{\mathrm{sc}}\right)$ and a high FF of $>0.6$ in the device.
Quantitative energy-level offsets in operando BHJ devices. To further diagnose the effects of the materials and the device configuration on the device performance, it is extremely important to obtain quantitative information on the energy band alignment. However, careful analysis reveals that the energy-level offsets directly obtained from the data shown in Fig. $2 \mathrm{~g}$ are smaller than that expected from the design and performance of the device. For example, the reduction in $V_{\mathrm{td}}$ in an open-circuit configuration under illumination from that in the dark state should be equal to the device $V_{\text {oc }}(0.61 \mathrm{~V})$; however, a much smaller value $(0.25 \mathrm{~V})$ is found in the SKPM measurement. Furthermore, the energy band diagram in Fig. 3 shows that the $V_{\text {td }}$ in the dark state should be equal to the difference in the work function of the electrodes of $1.6 \mathrm{~V}$ (the work functions of the $\mathrm{MoO}_{x} / \mathrm{ITO}$ and $\mathrm{LiF} / \mathrm{Al}$ electrodes have been separately measured to be 5.3 and $3.7 \mathrm{eV}$ using ultraviolet photon spectroscopy (UPS) and a dark $J-V$ fitting method on the basis of a diode model, respectively) ${ }^{14,39}$. However, the SKPM-measured value is $0.50 \mathrm{~V}$ (Fig. $2 \mathrm{~g}$ ). This discrepancy in the quantitative values has previously been encountered in studies on device cross-sections ${ }^{21-23}$ and has become a major obstacle in analysing device performance using the cross-sectional SKPM potentiometry.

We suspect that these results originate from systematic artefacts in SKPM including the finite-size tip convolution effect and the cantilever beam crosstalk. SKPM exploits long-range electrostatic forces; therefore, interaction between a large area of the sample and the entire tip/cantilever assembly (including the tip apex, the cone- or pyramidal-shaped tip and the cantilever beam) is probed (Fig. $4 a)^{34,40}$. These effects can be mathematically treated with the convolution of the true potential profile with a transfer function, which is dependent on the tip size, shape, tip-sample distance and so on, and is often modelled with a Gaussian function (Fig. $4 \mathrm{~b})^{32,41}$. If the width of the transfer function is much smaller than the sample feature size, the artefacts can be relatively minor (Supplementary Fig. 4); however, when the sample feature size is comparable to the tip size and tipsample distance, the effects of tip/cantilever convolution may cause an apparent smoothing and averaging in measured profiles (Fig. 4b $)^{42}$. Different methods such as the frequency-modulation mode SKPM ${ }^{32}$ and scan configuration optimization ${ }^{33}$ have been explored to reduce the artefacts in SKPM measurements; however, the effect of finite tip size and cantilever crosstalk cannot be completely eliminated. Since the cross-section of OPV devices is composed of multiple layers of different materials with a total thickness of $\sim 500 \mathrm{~nm}$ (the active layer is 200 -nm thick), which is comparable to the tip size $(\sim 20 \mathrm{~nm}$ in the tip apex radius and $\sim 10 \mu \mathrm{m}$ in tip height) and the average tip-sample distance in experiments (typically $10-\mathrm{nm}$ lift height plus $20-\mathrm{nm}$ 
tip oscillation amplitude), it is highly plausible that the tip/ cantilever convolution effects may drastically influence the SKPM potentiometry in our experiments.

The SP profiles of the device under different bias voltages ( $V_{\text {bias }}$ ) between the anode and cathode (Fig. 2h) further corroborate this hypothesis. In the experimental configuration shown in Fig. 1b, a forward $V_{\text {bias }}$ introduces an electric field in the same direction as the photo-induced electric field, which separates the quasi-Fermi levels and decreases $q V_{\mathrm{td}}$ (Fig. 3d); vice versa, a reverse $V_{\text {bias }}$ enlarges the total potential drop. Ideally, the $V_{\mathrm{td}}$ should decrease by $1.0 \mathrm{~V}$ under a forward $V_{\text {bias }}$ of $+1.0 \mathrm{~V}$; however, the apparent reduction in $V_{\text {td }}$ is only $0.41 \mathrm{~V}$, as shown in Fig. $2 \mathrm{~h}$. Similarly, the $V_{\mathrm{td}}$ should increase by $1.0 \mathrm{~V}$ under a reverse $V_{\text {bias }}$ of $-1.0 \mathrm{~V}$, but an increase in only $0.40 \mathrm{~V}$ is seen in Fig. $2 \mathrm{~h}$. The variation in $V_{\mathrm{td}}$ is plotted against $V_{\text {bias }}$ in Supplementary Fig. 5. A good linear correlation is found for the curve; however, the slope is considerably less than $1(\simeq 0.41)$, confirming the existence of a systematic and severe underestimate of SP differences in these measurements.
Interestingly, a quantitative correlation is observed between the $J-V$ performance of the OPV device and SKPM measurements. The SP profile obtained under a forward $V_{\text {bias }}$ of $+0.60 \mathrm{~V}$ appears to be identical to the SP profiles in open-circuit under illumination (Fig. 5a, the original SP images are shown in Supplementary Fig. 6). The overlay of the two measured profiles means that the actual profiles would also be identical if they had been deconvoluted (Fig. 5b). That is to say, the $V_{\mathrm{oc}}$ measured from SKPM is $0.6 \mathrm{~V}$, which is well agreed with the $0.61 \mathrm{~V}$ from device $J-V$ measurements.

It is not a simple coincidence that a bias voltage equal to $V_{\mathrm{oc}}$ is required to overlay the SP profile in the dark to that under illumination. The same result is observed for P3HT:indene- $\mathrm{C}_{60}$ bisadduct (ICBA) BHJ devices (Fig. 5a), which have the same device structure as the aforementioned P3HT:PCBM BHJ devices except that a different acceptor material is used, ICBA. The fabrication of the P3HT:ICBA device is described in detail in the Methods section. Figure $5 \mathrm{a}$ shows that the SP profiles of PCBM and ICBA devices are almost identical in the dark, but that the $V_{\mathrm{td}}$ a

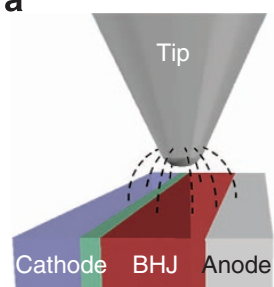

b

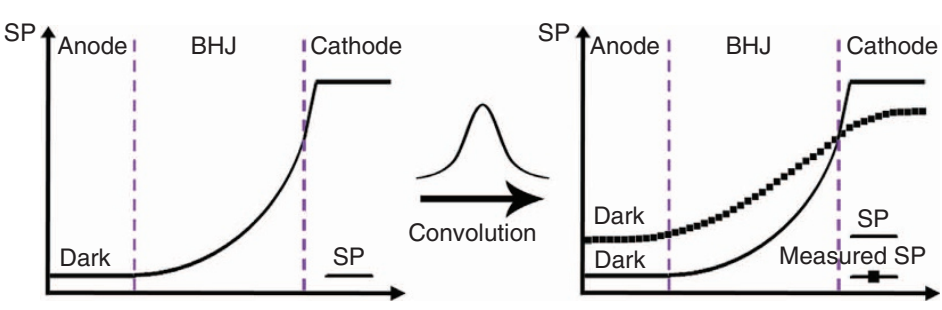

Figure 4 | The tip-induced averaging effect in SKPM measurements. (a) Schematic illustration of a finite-sized SKPM tip scanning over a cross-section of a multilayered thin-film device; (b) an illustration of the measured SP profile resulted from the convolution of the true profile with the transfer function of the SKPM tip.
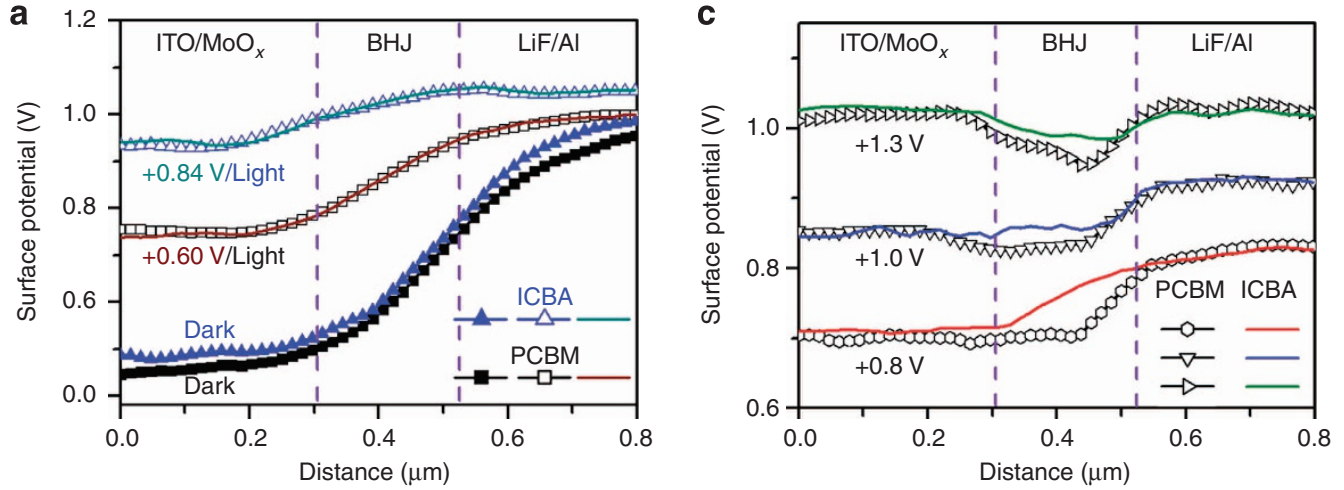

b

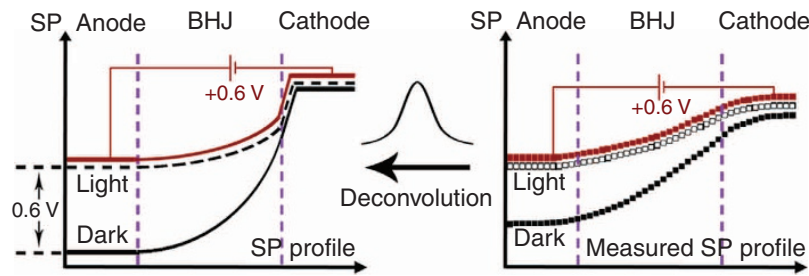

d

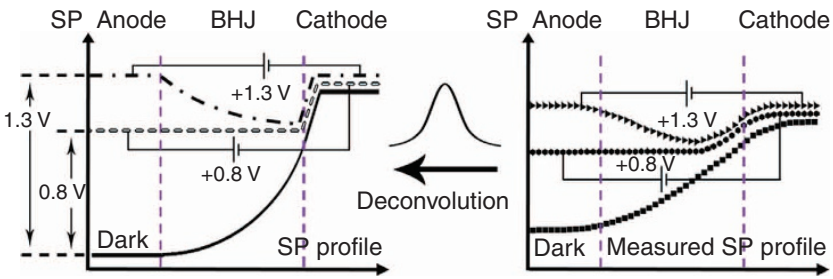

Figure 5 | Quantification of energy-level offsets by using the bias compensation method. (a) SP depth profiles of ITO/MoO $/ \mathrm{P3HT:acceptor/LiF/AI}$ devices with PCBM or ICBA acceptors in the dark, under AM $1.5 \mathrm{G}$ illumination and at biased conditions $(0.60$ and $0.84 \mathrm{~V}$ forward bias on the PCBM and ICBA devices, respectively). (b) Illustration of the quantitative measurement of $V_{\text {oc }}$ using the data from P3HT:PCBM device as an example. Overlapping SP profiles in SKPM measurements means that the actual profiles are also identical if they had been deconvoluted. (c) SP depth profiles of the two devices in the dark with different bias voltages of $+0.8,+1.0$ and $+1.3 \mathrm{~V}$. (d) Illustration of the bias compensation method using the data from the P3HT:PCBM device as an example. Flat-band and levelled electrode conditions would remain if the measured profiles could be deconvoluted. 
is reduced by $0.38 \mathrm{~V}$ for the ICBA device on illumination, which is considerably larger than the corresponding reduction of $0.25 \mathrm{~V}$ for the PCBM device. A $V_{\text {bias }}$ of $+0.84 \mathrm{~V}$ is required to overlay the SP profile with that of the P3HT:ICBA device under illumination (the original SP images are shown in Supplementary Fig. 7), which is equal to the $V_{\text {oc }}$ that is obtained from the $J-V$ characteristics (Supplementary Fig. 8). The higher $V_{\mathrm{oc}}$ value for the ICBA device compared with that of the PCBM device is in good agreement with reports in the literature (detailed parameters of the ICBA device before and after cross-section preparation and after SKPM measurement are in Supplementary Fig. 9 and Table 1$)^{43}$.

The accurate measurement of $V_{\text {oc }}$ indicates that SKPM potentiometry can yield quantitative information in spite of the presence of severe tip/cantilever convolution effect. The external bias between the anode and cathode plays an important role in this process and can be further exploited for the quantitative measurements of other energy-level offsets in device depth profiles. For example, the measured profile in Fig. $2 \mathrm{~g}$ is strongly influenced by the convolution effect: the potential value along the line is smoothed and averaged, leading to an underestimate of potential differences between points along the profile. To obtain the true SP difference between two points, for example, the cathode and the anode, we may apply an external bias between the electrodes and look for the bias voltage required to equalize the SP of the two electrodes. When the SP difference is fully compensated by the external bias, the two electrodes are at the same potential. At this point, the effect of smoothing and averaging due to tip/cantilever convolution is minimized, and the external bias voltage reflects the true SP difference (Fig. $5 \mathrm{~d}$ ). It is emphasized that this bias compensation method does not reduce or remove the tip/cantilever convolution effect, but, instead, this method seeks to create an equal potential condition where the effect of convolution is minimized, such that the true SP difference between certain energy levels can be probed.

Figure $5 \mathrm{c}$ shows the measurement of $V_{\text {td }}$ in the P3HT:PCBM and P3HT:ICBA BHJ devices using the bias compensation method. $V_{\mathrm{td}}$ is an important parameter in the energy band alignment. As explained in Fig. 3b, the value of $q V_{\mathrm{td}}$ equals the sum of $q V_{\mathrm{bi}}$ and the cathode interface VL shift $(\Delta)$; thus, $V_{\mathrm{td}}$ is taken to be the upper limit of $V_{\mathrm{bi}}$. In principle, $\mathrm{q} V_{\mathrm{td}}$ can be obtained by separate measurements of the work function of the two electrodes using UPS; however, the low work function cathode material is often partially oxidized ${ }^{44}$, and thus the $V_{\mathrm{td}}$ has not been measured previously in operating OPV devices.

In the measurement of $V_{\mathrm{td}}$ via the bias compensation method, the external bias necessary to equalize the SP of the two electrodes is searched. Since the experiments in Fig. $2 \mathrm{~h}$ have established a monotonic correlation between the applied bias and the reduction in SP difference between the electrodes, it is thus natural to further raise the bias to equalize the electrode potentials. Figure $5 \mathrm{c}$ shows that when the forward $V_{\text {bias }}$ is increased to $+1.3 \mathrm{~V}$, both the PCBM and ICBA devices show equal SPs between the cathode and the anode. This result means that the work function difference between the two electrodes is compensated by the $1.3 \mathrm{~V}$ forward $V_{\text {bias. }}$. It confirms that the overall $q V_{\text {td }}$ is determined by the electrode materials and is independent of the active material. The corresponding energy band diagram of the cathode-anode VL alignment condition is shown in Fig. 6a. In addition, under a $V_{\text {bias }}$ of $1.3 \mathrm{~V}$, the active layer in PCBM shows higher reversed band bending and a larger SP difference between the cathode and the active layer than the ICBA, which is selfconsistent with the cathode interfacial dipole of the ICBA being stronger than that of the PCBM. The difference between the work function for the $\mathrm{MoO}_{x} / \mathrm{ITO}$ and $\mathrm{LiF} / \mathrm{Al}$ electrodes has never been directly measured in operando devices. The measured value of
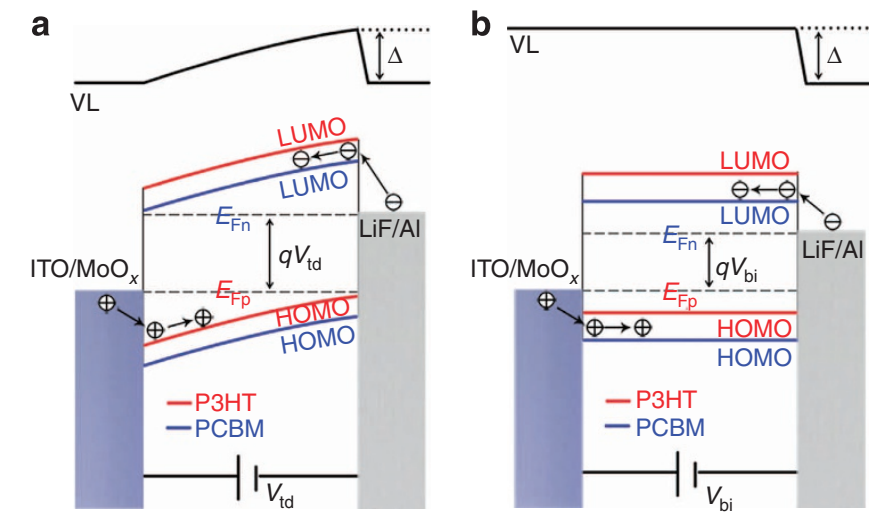

Figure 6 | Energy band diagrams under different bias conditions. A ITO/ $\mathrm{MoO}_{x} / \mathrm{P} 3 \mathrm{HT}: \mathrm{PCBM} / \mathrm{LiF} / \mathrm{Al}$ device under (a) cathode-anode VL alignment condition and (b) active layer flat-band condition.

$1.3 \mathrm{eV}$ using the bias compensation method is reasonable and fairly close to the value of $1.6 \mathrm{~V}$ derived from the difference between the work function of $\mathrm{MoO}_{x} / \mathrm{ITO}$ and $\mathrm{LiF} / \mathrm{Al}$ electrodes, as measured by UPS and the diode-model-based dark $J-V$ fitting method, respectively ${ }^{14,39}$.

Very importantly, the $V_{\mathrm{bi}}$ of BHJ OPV devices can also be measured using the bias compensation method in SKPM. Accurate measurement of $V_{\mathrm{bi}}$ is critical for understanding the device mechanism because $V_{\mathrm{bi}}$ strongly affects charge separation, transport, collection and recombination and is the upper limit of the device $V_{\text {oc }}$ (refs 14,45). In $\mathrm{p}-\mathrm{n}$ junction diodes, the $V_{\mathrm{bi}}$ is defined as the potential difference across the two ends of the $\mathrm{p}-\mathrm{n}$ junction, but the $V_{\mathrm{bi}}$ in a BHJ OPV is difficult to define and measure because the diode junction contains a phase-separated bi-continuous mixture. If we apply the classic metal-insulatormetal model to the OPV, the value of $q V_{\mathrm{bi}}$ equals the difference in the work function between the anode and the cathode, which manifests as $q V_{\mathrm{td}}$ in the operando device ${ }^{45}$. However, this approximation for $V_{\mathrm{bi}}$ completely neglects molecular details and interfacial states, and thus cannot accurately account for the behaviour of the device.

If we define the $V_{\mathrm{bi}}$ in an OPV similarly to that in $\mathrm{p}-\mathrm{n}$ junction diodes, that is, as the potential difference across the two ends of the $\mathrm{BHJ}$, the $V_{\mathrm{bi}}$ is practically equal to the $V_{\text {bias }}$ that is required to level the potential of the two ends of the BHJ, that is, the flat-band potential $V_{\mathrm{fb}}$, in light of the bias compensation method (the corresponding energy band diagram is illustrated in Fig. 6b). Figure $5 c$ shows the measurement of $V_{\mathrm{fb}}$ or $V_{\mathrm{bi}}$ of the PCBM and ICBA devices. Under a $+0.8 \mathrm{~V}$ forward $V_{\text {bias }}$, the SP profile from the anode to the most part of the active layer in the PCBM device is flat, and the only potential drop occurs at the cathodic interface (because of the interfacial dipole) ${ }^{7}$. Thus, we infer that the $V_{\mathrm{bi}}$ of the P3HT:PCBM device is $\simeq 0.8 \mathrm{~V}$. Taking the active layer thickness to be $\simeq 200 \mathrm{~nm}$, the built-in field is estimated to be $\sim 4 \times 10^{4} \mathrm{~V} \mathrm{~cm}^{-1}$, which falls within the $\simeq 1-10 \times 10^{4} \mathrm{~V} \mathrm{~cm}^{-1}$ range that was estimated in a previous publication ${ }^{19}$ and is close to the $8 \times 10^{4} \mathrm{~V} \mathrm{~cm}^{-1}$ value that was obtained in a numerical simulation on the basis of the Blom model ${ }^{46}$ and the Nelson model $^{47}$. In contrast, the ICBA device under $+0.8 \mathrm{~V} V_{\text {bias }}$ still exhibits a continuous potential drop and significant band bending. A $V_{\text {bias }}$ of $+1.0 \mathrm{~V}$ is required to reach the flat-band condition in the ICBA device; therefore, the $V_{\mathrm{bi}}$ for the P3HT:ICBA device is $\simeq 1.0 \mathrm{~V}$. Higher $V_{\mathrm{bi}}$ in the ICBA device than that in the PCBM device is consistent with Mott-Schottky analysis (Supplementary Fig. 10). Both the $0.8 \mathrm{~V}$ value in the PCBM device and the $1.0 \mathrm{~V}$ value in the ICBA device obtained via 
the bias compensation method are greater than the device $V_{\text {oc }}$, which is consistent with concept that $V_{\mathrm{bi}}$ is the upper limit of $V_{\mathrm{oc}}$, and therefore shall be greater than it ${ }^{14,45}$.

Thus, we have demonstrated that important parameters such as $V_{\mathrm{oc}}, V_{\mathrm{td}}$ and $V_{\mathrm{bi}}$, can be quantitatively measured using the bias compensation method in spite of the presence of the tip/ cantilever convolution effect in SKPM. This breakthrough is generally valuable for SKPM and particularly intriguing for mechanistic studies on OPVs and other thin-film devices.

Energy band alignment in planar junction devices. To provide another example of the correlation between the SP profile and the device performance, we investigate a planar junction (PJ) device using P3HT- and PCBM-active materials. The preparation of the PJ device is described in detail in the Methods section. It is critical to avoid thermal annealing to minimize interlayer diffusion and obtain an abrupt donor-acceptor junction/interface between the $\mathrm{P} 3 \mathrm{HT}$ and the $\mathrm{PCBM}^{48}$. Figure 7 a shows the $J-V$ characteristics of the ITO/ZnO/PCBM/P3HT/ $/ \mathrm{MoO}_{x} / \mathrm{Al} \mathrm{PJ}$ device. The $V_{\text {oc }}(0.61 \mathrm{~V})$ and $\mathrm{FF}(0.61)$ of the PJ device are comparable to those of the $\mathrm{BHJ}$ device in Fig. 1; however, the $J_{\mathrm{sc}}\left(1.01 \mathrm{~mA} \mathrm{~cm}^{-2}\right)$ is much lower than that of the $\mathrm{BHJ}$ device. The PJ device also shows much lower external quantum efficiency (EQE) than the $\mathrm{BHJ}$ device (Fig. 7b), and the peak in EQE does not overlap with the absorption of active materials but severely leans towards the short-wavelength region. The cross-sectional SP image and the depth profile of the PJ device under short-circuit conditions in the dark (Fig. 7c,d) show that the majority of potential drop occurs at the interface between the P3HT and the PCBM and that there are regions in the $\mathrm{P} 3 \mathrm{HT}$ and PCBM layers where the potential change is rather small and the electric field is very weak, which is very different from the $\mathrm{BHJ}$ case for which a continuous potential drop is observed across the entire $\mathrm{BHJ}$ layer. The fact that the VL does not align at the organic/organic, that is, $\mathrm{P} 3 \mathrm{HT} / \mathrm{PCBM}$, interface is consistent with the UPS results ${ }^{49}$. Spontaneous electron transfer has been reported from the donor P3HT to the acceptor PCBM, which results in an interface dipole with its positive end towards the P3HT layer and its negative end towards the PCBM layer ${ }^{50}$. The SP depth profile in Fig. $7 \mathrm{~d}$ is in qualitative agreement with this picture.
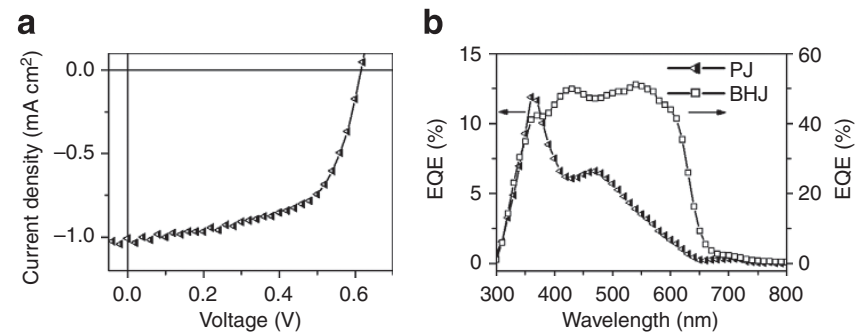

C

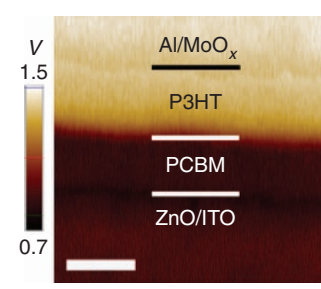

\section{d}

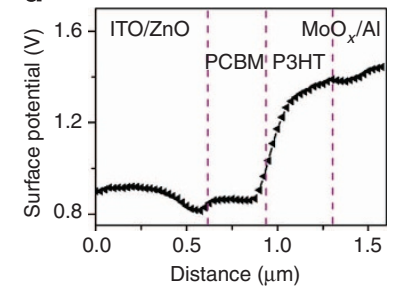

Figure 7 | Device characteristics and SP of a PJ device. (a) $J-V$ curve of a ITO/ZnO/PCBM/P3HT/MoO $/$ Al PJ device under AM 1.5G illumination, (b) EQE of the PJ device (triangles) and the $\mathrm{BHJ}$ device (squares) that are shown in Fig. 2, (c) SP image (scale bar, $400 \mathrm{~nm}$ ) and (d) SP profile of the PJ device cross-section under short-circuit conditions in the dark.

\section{Discussion}

This contribution shows the realization of operando mapping of the energy band depth profile in OPV devices. A bias compensation method has been devised to measure energy-level offsets. This study clearly indicates that the tip/cantilever convolution effect and the associated low spatial resolution in SKPM constitute major bottlenecks in unveiling the true energy band alignment profile. Ideally, deconvoluted energy profiles with nanometre or subnanometre spatial resolution could lead to accurate calculation of the device internal field and spatially resolved understanding of the drift current within the device. While technical advances such as frequency modulation-SKPM and/or optimized scan configuration may improve the spatial resolution on the current study, ultimately, future efforts in reducing the tip size and hence actual reduction in the tip convolution effects will be highly desirable for mechanistic understanding of thin-film devices.

In conclusion, we have directly visualized energy band alignment in operating OPV devices using cross-sectional SKPM imaging. A bias compensation method allows for quantitative measurements of energy-level differences such as $V_{\mathrm{oc}}, V_{\mathrm{bi}}$ and $V_{\mathrm{td}}$ in operating OPV devices. The resulting SP profiles of $\mathrm{BHJ}$ and $\mathrm{PJ}$ P3HT:PCBM devices show that a continuous potential drop across the entire $\mathrm{BHJ}$-active layer is critical to facilitate carrier separation and collection for high $J_{\mathrm{sc}}$ and $\mathrm{FF}$ in device performance, whereas the donor-acceptor interface dipole efficiently promotes the dissociation of photo-generated excitons. This approach should be generally applicable to other thin-film devices, such as perovskite and quantum dot solar cells, lightemitting diodes and thin-film transistors, and could contribute significantly to the understanding of the mechanisms in and improvements of these devices.

\section{Methods}

Materials. All of the active layer materials are purchased and used as received. The P3HT is obtained from 1-Material Inc., the PCBM is obtained from American Dye Source Inc. and the ICBA is obtained from Solenne BV.

BHJ device preparation. The $\mathrm{BHJ}$ device is fabricated by sequentially cleaning pre-patterned ITO glass substrates $\left(15 \Omega \mathrm{sq}^{-1}\right)$ using ultrasonication for $10 \mathrm{~min}$ in a detergent, deionized water, ethanol, acetone and isopropyl alcohol, followed by treatment with oxygen plasma for $10 \mathrm{~min}$. Then, a $10-\mathrm{nm} \mathrm{MoO}_{x}$ layer, which serves as an anode interlayer, is thermally evaporated on ITO substrates under a pressure of $4 \times 10^{-4} \mathrm{~Pa}$. The active layer consists of different blends that are prepared by spin-coating, followed by being subjected to different annealing conditions. A 1-nm $\mathrm{LiF}$ layer and a $100-\mathrm{nm} \mathrm{Al}$ layer are subsequently evaporated under a pressure of $4 \times 10^{-4} \mathrm{~Pa}$ through a shadow mask to define the active area of the devices $\left(0.12 \mathrm{~cm}^{2}\right)$ and to form a top cathode. P3HT:PCBM $\left(36 \mathrm{mg} \mathrm{ml}^{-1}, 1: 0.8 \mathrm{w} / \mathrm{w}\right)$ and P3HT:ICBA (34 $\mathrm{mg} \mathrm{ml}^{-1}, 1: 1 \mathrm{w} / \mathrm{w}$ ) are dissolved in 1,2-dichlorobenzene. Both blends are stirred at $60^{\circ} \mathrm{C}$ for $\simeq 14 \mathrm{~h}$ in a glove box. P3HT:PCBM is spin-coated at 600 r.p.m. for $60 \mathrm{~s}$, followed by pre-annealing at $130^{\circ} \mathrm{C}$ for $10 \mathrm{~min}$. After spincoating at 600 r.p.m. for $20 \mathrm{~s}$, the wet P3HT:ICBA blend film is subjected to solvent-annealing for $\simeq 30 \mathrm{~min}$, followed by post annealing at $150{ }^{\circ} \mathrm{C}$ for $10 \mathrm{~min}$.

PJ device preparation. The PJ device is fabricated by floating a P3HT film on a PCBM film. PCBM and P3HT are both dissolved in chlorobenzene to form a 20 - $\mathrm{mg} \mathrm{ml}^{-1}$ solution. The PCBM film is spin-coated on a $\mathrm{ZnO}$ nanocrystal ${ }^{51,52}$ modified pre-patterned ITO substrate, followed by thermal annealing at $130^{\circ} \mathrm{C}$ for 2 min. The P3HT film is spin-coated on bare glass and, following thermal annealing at $130^{\circ} \mathrm{C}$ for $10 \mathrm{~min}$, the film is suspended over a hydrofluoric acid (HF) solution for $1 \mathrm{~min}$ and floated in deionized water. After the PCBM-film-coated $\mathrm{ZnO} / \mathrm{ITO}$ substrate picks up the free-standing P3HT film, the substrate is placed in a vacuum oven $(\simeq 1 \mathrm{~Pa})$ for $\sim 1$ h to improve the contact between the bilayer films. Then, a $10-\mathrm{nm} \mathrm{MoO}_{x}$ layer and a $100-\mathrm{nm}$ Al layer are subsequently evaporated under a pressure of $4 \times 10^{-4} \mathrm{~Pa}$ through a shadow mask to define the active area of the devices $\left(0.12 \mathrm{~cm}^{2}\right)$ and to form a top electrode.

Device characterization. The $J-V$ characteristics of the devices are recorded using a Keithley 2635A sourcemeter (Keithley Instruments Inc., Cleveland, OH, USA) or an Agilent B1500A-SDA (Agilent Technologies Inc., Santa Clara, CA, USA) under one sun, AM 1.5G irradiation $\left(100 \mathrm{~mW} \mathrm{~cm}^{-2}\right)$ from a solar simulator (Newport 
67005, Newport, Irvine, CA, USA). The illumination intensity is calibrated using a reference cell (Oriel 91150V). EQE spectra are measured by a Merlin (Model No. 70104) Digital Lock-in Radiometry System using a 300-W xenon lamp and a 74125 Oriel Cornerstone $260 \frac{1}{4}-\mathrm{m}$ monochromator with order-sorting filters (Newport). The light intensity at different wavelengths is modulated through an aperture and calibrated using a 70714 UV-enhanced Si photodiode (Newport). All of the measurements are performed under ambient atmosphere at room temperature.

Device cross-section preparation. The device cross-section is fabricated using an Ilion ${ }^{+} 693$ System (Gatan Inc.). A freshly mechanically cleaved device is mounted into the vacuum chamber $\left(6.4 \times 10^{-5}\right.$ Torr $)$ and cooled using liquid nitrogen. Then, the device is milled by argon ions using a beam voltage of $5 \mathrm{keV}$ and a beam current of $10 \mu \mathrm{A}$ for $\simeq 2 \mathrm{~h}$. Four to five cross-section samples are prepared for each device under study. The $J-V$ curves and parameter statistics before and after crosssection preparation are shown in Supplementary Figs 11-13 and Supplementary Tables 1-3.

A control experiment was carried out to examine the ion-beam damage in different materials. As shown in Supplementary Fig. 14, P3HT, PCBM and P3HT:PCBM BHJ thin-film samples are subjected to different time of ion milling, and then imaged with SKPM. The general trend is that the longer ion-milling time, the more material was etched away, and the more significantly the SP changed. The reversal of the SP change as the film became almost completely etched is probably due to the influence of the substrates. These results indicate that the polymer materials are indeed damaged under $\mathrm{Ar}^{+}$ion beam, but freshly exposed surfaces (the shortest time) exhibit similar SP changes compared with respective pristine films $(\sim 50,30$ and $60 \mathrm{mV}$ for P3HT, PCBM and P3HT:PCBM thin films, respectively); therefore, the relative SP difference values are reliable. Consider the ion-milling configuration in cross-section preparation (Supplementary Fig. 15), the cross-section surface finally exposed for SKPM measurements can be considered fresh surface since majority of etching and damage happens at the frontier of ion beam rather than the sideway.

Device cross-section characterization. The morphology of the device crosssection is characterized using FEI Quanta 400 FEG SEM (FEI Corp., Hillsboro, OR, USA). The SKPM SP profile measurements of device cross-sections are carried out using a Park XE-120 AFM (Park Systems Corp., Suwon, Korea) using Ti/Pt-coated conducting tips (PPP-EFM, Nanosensor, Neuchatel, Switzerland) with a resonance frequency of $\sim 80 \mathrm{kHz}$ and a spring constant of $\sim 0.6 \mathrm{~N} \mathrm{~m}^{-1}$. A two-pass scan AM mode SKPM is used to measure the SP under an environment with relative humidity below $20 \%$. During the first pass, standard AC mode imaging (typical tip oscillation amplitude $20 \mathrm{~nm}$ ) is performed to acquire the topography and phase signal of the sample; in the second pass, the tip is lifted up by a certain height (typically $10 \mathrm{~nm}$ ) and scanned on the basis of the topography line obtained from the first pass. An AC voltage ( $3 \mathrm{~V}$ in amplitude and $10 \mathrm{kHz}$ in frequency) is applied to actuate the cantilever, and the DC voltage applied to the tip that nullifies the tipsample interaction is collected as the SP signal. The device-wiring configuration during cross-section characterization is shown in Fig. 1b. The Al electrode is grounded. The ITO electrode is directly connected with the Al electrode in shortcircuit; open-circuit conditions were simulated by disconnecting the wire between two electrodes. A full-spectrum optical fibre is used to transmit AM $1.5 \mathrm{G}$ solar simulator light to illuminate the device from the ITO glass side. Device forward or reverse bias voltage was applied via a tuneable voltage source between the electrodes.

\section{References}

1. Green, M. A. Solar Cells: Operating Principles, Technology, and System Applications (Prentice-Hall, 1982).

2. Veldman, D. et al. Compositional and electric field dependence of the dissociation of charge transfer excitons in alternating polyfluorene copolymer/ fullerene blends. J. Am. Chem. Soc. 130, 7721-7735 (2008).

3. Mozer, A. J. et al. Time-dependent mobility and recombination of the photoinduced charge carriers in conjugated polymer/fullerene bulk heterojunction solar cells. Phys. Rev. B 72, 035217 (2005).

4. Street, R. A., Cowan, S. \& Heeger, A. J. Experimental test for geminate recombination applied to organic solar cells. Phys. Rev. B 82, 121301(R) (2010).

5. Mihailetchi, V. D., Koster, L. J. A., Hummelen, J. C. \& Blom, P. W. M. Photocurrent generation in polymer-fullerene bulk heterojunctions. Phys. Rev. Lett. 93, 216601 (2004).

6. Blom, P. W. M., Mihailetchi, V. D., Koster, L. J. A. \& Markov, D. E. Device physics of polymer : fullerene bulk heterojunction solar cells. Adv. Mater. 19, 1551-1566 (2007).

7. Guerrero, A. et al. How the charge-neutrality level of interface states controls energy level alignment in cathode contacts of organic bulk-heterojunction solar cells. ACS Nano 6, 3453-3460 (2012).

8. Guerrero, A. et al. Interplay between fullerene surface coverage and contact selectivity of cathode interfaces in organic solar cells. ACS Nano 7, 4637-4646 (2013).
9. Zhou, H. Q. et al. High-efficiency polymer solar cells enhanced by solvent treatment. Adv. Mater. 25, 1646-1652 (2013).

10. Hegedus, S. S. \& Shafarman, W. N. Thin-film solar cells: device measurements and analysis. Prog. Photovolt Res. Appl. 12, 155-176 (2004).

11. Wu, H., Huang, F., Peng, J. \& Cao, Y. High-efficiency electron injection cathode of Au for polymer light-emitting devices. Org. Electron. 6, 118-128 (2005).

12. MacLeod, B. A. et al. Built-in potential in conjugated polymer diodes with changing anode work function: interfacial states and deviation from the Schottky-Mott limit. J. Phys. Chem. Lett. 3, 1202-1207 (2012).

13. Zimmermann, B., Glatthaar, M., Niggemann, M., Riede, M. \& Hinsch, A. Electroabsorption studies of organic bulk-heterojunction solar cells. Thin Solid Films 493, 170-174 (2005).

14. Mihailetchi, V. D., Blom, P. W. M., Hummelen, J. C. \& Rispens, M. T. Cathode dependence of the open-circuit voltage of polymer: fullerene bulk heterojunction solar cells. J. Appl. Phys. 94, 6849-6854 (2003).

15. Ishii, H. et al. Kelvin probe study of band bending at organic semiconductor/ metal interfaces: examination of Fermi level alignment. Phys. Stat. Sol. A 201, 1075-1094 (2004).

16. Pingree, L. S. C., Reid, O. G. \& Ginger, D. S. Electrical scanning probe microscopy on active organic electronic devices. Adv. Mater. 21, 19-28 (2009).

17. Hoppe, H. et al. Kelvin probe force microscopy study on conjugated polymer/ fullerene bulk heterojunction organic solar cells. Nano Lett. 5, 269-274 (2005).

18. Chen, F. et al. Tuning indium tin oxide work function with solution-processed alkali carbonate interfacial layers for high-efficiency inverted organic photovoltaic cells. Nanotechnology 24, 484011 (2013).

19. Yuan, Y. B. et al. Efficiency enhancement in organic solar cells with ferroelectric polymers. Nat. Mater. 10, 296-302 (2011).

20. He, Z. C. et al. Simultaneous enhancement of open-circuit voltage, short-circuit current density, and fill factor in polymer solar cells. Adv. Mater. 23, 4636-4643 (2011).

21. Lee, J., Kong, J., Kim, H., Kang, S.-O. \& Lee, K. Direct observation of internal potential distributions in a bulk heterojunction solar cell. Appl. Phys. Lett. 99, 243301 (2011).

22. Kong, J. et al. Biased internal potential distributions in a bulk-heterojunction organic solar cell incorporated with a TiOx interlayer. Appl. Phys. Lett. 100, 213305 (2012).

23. Saive, R. et al. Imaging the electric potential within organic solar cells. Adv. Funct. Mater. 23, 5854-5860 (2013).

24. Bergmann, V. W. et al. Real-space observation of unbalanced charge distribution inside a perovskite-sensitized solar cell. Nat. Commun. 5, 5001 (2014).

25. Glatzel, T., Marron, D. F., Schedel-Niedrig, T., Sadewasser, S. \& Lux-Steiner, M. C. CuGaSe2 solar cell cross section studied by Kelvin probe force microscopy in ultrahigh vacuum. Appl. Phys. Lett. 81, 2017-2019 (2002).

26. Jiang, C.-S., Moutinho, H. R., Friedman, D. J., Geisz, J. F. \& Al-Jassim, M. M. Measurement of built-in electrical potential in III-V solar cells by scanning Kelvin probe microscopy. J. Appl. Phys. 93, 10035-10040 (2003).

27. Ishii, H., Sugiyama, K., Ito, E. \& Seki, K. Energy level alignment and interfacial electronic structures at organic metal and organic organic interfaces. Adv. Mater. 11, 605-625 (1999).

28. Cahen, D. \& Kahn, A. Electron energetics at surfaces and interfaces: concepts and experiments. Adv. Mater. 15, 271-277 (2003).

29. He, Z. C. et al. Enhanced power-conversion efficiency in polymer solar cells using an inverted device structure. Nat. Photon 6, 591-595 (2012).

30. Mao, L. et al. Flexible silver grid/PEDOT:PSS hybrid electrodes for large area inverted polymer solar cells. Nano Energy 10, 259-267 (2014).

31 . You, J. et al. A polymer tandem solar cell with $10.6 \%$ power conversion efficiency. Nat. Commun. 4, 1446 (2013).

32. Cohen, G. et al. Reconstruction of surface potential from Kelvin probe force microscopy images. Nanotechnology 24, 295702 (2013).

33. Charrier, D. S. H., Kemerink, M., Smalbrugge, B. E., de Vries, T. \& Janssen, R. A. J. Real versus measured surface potentials in scanning Kelvin probe microscopy. ACS Nano 2, 622-626 (2008).

34. Jacobs, H. O., Leuchtmann, P., Homan, O. J. \& Stemmer, A. Resolution and contrast in Kelvin probe force microscopy. J. Appl. Phys. 84, 1168-1173 (1998)

35. Jorgensen, M., Norrman, K. \& Krebs, F. C. Stability/degradation of polymer solar cells. Sol. Energy Mater. Sol. C 92, 686-714 (2008).

36. Vasilopoulou, M. et al. Large work function shift of organic semiconductors inducing enhanced interfacial electron transfer in organic optoelectronics enabled by porphyrin aggregated nanostructures. Nano Res. 7, 679-693 (2014).

37. Campoy-Quiles, M. et al. Morphology evolution via self-organization and lateral and vertical diffusion in polymer: fullerene solar cell blends. Nat. Mater 7, 158-164 (2008).

38. Chen, D. A., Nakahara, A, Wei, D. G., Nordlund, D. \& Russell, T. P. P3HT/ PCBM bulk heterojunction organic photovoltaics: correlating efficiency and morphology. Nano Lett. 11, 561-567 (2011).

39. Shrotriya, V., Li, G., Yao, Y., Chu, C. W. \& Yang, Y. Transition metal oxides as the buffer layer for polymer photovoltaic cells. Appl. Phys. Lett. 88, 073508 (2006). 
40. Glatzel, T., Sadewasser, S. \& Lux-Steiner, M. C. Amplitude or frequency modulation-detection in Kelvin probe force microscopy. Appl. Surf. Sci. 210, 84-89 (2003).

41. Strassburg, E., Boag, A. \& Rosenwaks, Y. Reconstruction of electrostatic force microscopy images. Rev. Sci. Instrum. 76, 083705 (2005).

42. Liscio, A., Palermo, V. \& Samori, P. Nanoscale quantitative measurement of the potential of charged nanostructures by electrostatic and kelvin probe force microscopy: unraveling electronic processes in complex materials. Accounts Chem. Res. 43, 541-550 (2010).

43. He, Y., Chen, H.-Y., Hou, J. \& Li, Y. Indene-C60 bisadduct: a new acceptor for high-performance polymer solar cells. J. Am. Chem. Soc. 132, 1377-1382 (2010).

44. Yip, H. L. \& Jen, A. K. Y. Recent advances in solution-processed interfacial materials for efficient and stable polymer solar cells. Energy Environ. Sci. 5, 5994-6011 (2012)

45. Malliaras, G. G., Salem, J. R., Brock, P. J. \& Scott, J. C. Photovoltaic measurement of the built-in potential in organic light emitting diodes and photodiodes. J. Appl. Phys. 84, 1583-1587 (1998).

46. Koster, L. J. A., Smits, E. C. P., Mihailetchi, V. D. \& Blom, P. W. M. Device model for the operation of polymer/fullerene bulk heterojunction solar cells. Phys. Rev. B 72, 085205 (2005).

47. Shuttle, C. G., Hamilton, R., O'Regan, B. C., Nelson, J. \& Durrant, J. R. Chargedensity-based analysis of the current-voltage response of polythiophene/fullerene photovoltaic devices. Proc. Natl Acad. Sci. USA 107, 16448-16452 (2010).

48. Treat, N. D. et al. Interdiffusion of PCBM and P3HT reveals miscibility in a photovoltaically active blend. Adv. Energy Mater. 1, 82-89 (2011).

49. Guan, Z. L. et al. Direct determination of the electronic structure of the poly(3-hexylthiophene):phenyl- 6,6 -C61 butyric acid methyl ester blend. Org. Electron. 11, 1779-1785 (2010).

50. Aarnio, H. et al. Spontaneous charge transfer and dipole formation at the interface between P3HT and PCBM. Adv. Energy Mater. 1, $792-797$ (2011).

51. Liang, X. et al. Colloidal indium-doped zinc oxide nanocrystals with tunable work function: rational synthesis and optoelectronic applications. Chem. Mater. 26, 5169-5178 (2014).

52. Bai, S. et al. High-performance planar heterojunction perovskite solar cells: preserving long charge carrier diffusion lengths and interfacial engineering. Nano Res. 7, 1749-1758 (2014).

\section{Acknowledgements}

We thank Professor Hongbin Wu at the South China University of Technology for a critical reading of the manuscript. Q.C. thanks Yi Zhang and Xiaodong Gao at the Electron Microscope Laboratory of SINANO, CAS for their assistance with fabricating the device cross-section and the SEM characterization. This work was supported by the National Natural Science Foundation of China (Grant Nos 91233104 and 51473184). Partial support from Collaborative Innovation Center of Suzhou Nano Science and Technology is also appreciated. L.C. acknowledges support from the Jiangsu Provincial Natural Science Foundation (Grant No. BK20130006).

\section{Author contributions}

L.C. and Q.C. developed the concept and designed the experiments. Q.C. performed the experiments; L.M., Y.L. and D.W. assisted with the device fabrication; W.L. assisted in SKPM characterization. L.C. supervised the data analysis. L.C., Q.C. and B.W. wrote the manuscript. N.W., C.M., S.B. and Y.J. provided the $\mathrm{ZnO}$ interlayer. T.K. assisted in the device $J-V$ measurements. All of the authors commented on the manuscript.

\section{Additional information}

Supplementary Information accompanies this paper at http://www.nature.com/ naturecommunications

Competing financial interests: The authors declare no competing financial interest.

Reprints and permission information is available online at http://npg.nature.com/ reprintsandpermissions/

How to cite this article: Chen, Q. et al. Quantitative operando visualization of the energy band depth profile in solar cells. Nat. Commun. 6:7745 doi: 10.1038/ncomms8745 (2015).

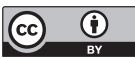

This work is licensed under a Creative Commons Attribution 4.0 International License. The images or other third party material in this article are included in the article's Creative Commons license, unless indicated otherwise in the credit line; if the material is not included under the Creative Commons license, users will need to obtain permission from the license holder to reproduce the material. To view a copy of this license, visit http://creativecommons.org/licenses/by/4.0/ 